\title{
Efeito da prática do Tai Chi Chuensobre a resistência aeróbia de idosas sedentárias
}

\author{
Effect of Tai Chi Chuan practice on aerobic resistance of sedentary elderly women
}

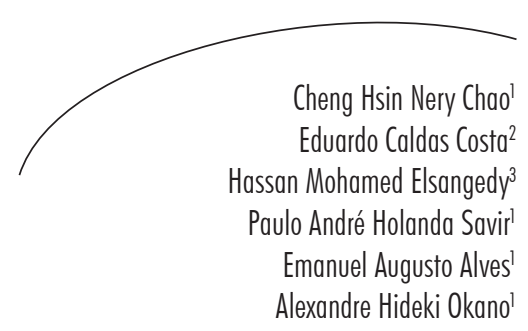

\section{Resumo}

Objetivo: Investigar o efeito da prática do tai chi chuan (TCC) sobre a resistência aeróbia de idosas sedentárias. Métodos: Fizeram parte do estudo 11 idosas sedentárias (67,9 \pm 6,8 anos; $25,9 \pm 2,8 \mathrm{~kg} / \mathrm{m}^{2}$ ), as quais foram submetidas aos testes de marcha estacionária de dois minutos e teste de caminhada de seis minutos pré e pós-intervenção de 12 semanas de TCC. Resultados: Houve aumento da resistência aeróbia das idosas analisadas após 12 semanas de prática de TCC, demonstrado tanto por meio do teste de caminhada de seis minutos $(510 \pm 64$ vs. $536 \pm 63$ metros; $p=0,006)$ quanto no teste de marcha estacionária de dois minutos (83 \pm 20 vs. $110 \pm 19$ execuções; $p=0,001)$. Conclusão: De acordo com os resultados obtidos, é possível concluir que a prática do TCC é capaz de promover aumento da resistência aeróbia de idosas sedentárias.

\section{Abstract}

Objective: To investigate the effect of tai chi chuan (TCC) practice on cardiorespiratory fitness of sedentary elderly women. Methods: A total of 11 sedentary elderly women (67.9 \pm 6.8 years; $25.9 \pm 2.8 \mathrm{~kg} \cdot \mathrm{m}^{-2}$ ) were submitted to six-minute walk test and two-minute step test before and after a 12-week TCC training program. Results: Improvements were observed on cardiorespiratory fitness of elderly women analyzed after a 12 -week TCC training program, through six-minute walk test $(510 \pm 64$ vs. $536 \pm 63$ meters; $p=$ $0.006)$ and two-minute step test $(83 \pm 20$ vs. $110 \pm 19$ executions; $p=0.001)$. Conclusion: According with obtained results, it is possible to conclude that the TCC practice may promote an increase in cardiorespiratory fitness in sedentary elderly women.

\footnotetext{
Departamento de Educação Física. Universidade Federal do Rio Grande do Norte. Natal, RN, Brasil.

2 Programa de Pós-graduação em Ciências da Saúde, Departamento de Educação Física. Universidade Federal do Rio Grande do Norte. Natal, RN, Brasil.

Programa de Pós-graduação em Educação Física. Universidade Federal do Paraná. Curitiba, PR, Brasil.
}

Palavras-chave: Tai chi chuan. Idoso. Resistência física. Testes de aptidão Aptidão física.

Key words: Tai chi chuan. Elderly. Physical fitness. Physical endurance. 


\section{INTRODUÇÃO}

Estudos transversais ${ }^{1,2}$ e longitudinais ${ }^{3,4}$ mostram que o envelhecimento gera perdas significativas na resistência aeróbia. Esse fato merece destaque, pois a redução da resistência aeróbia está associada ao aumento da dependência física dos idosos para realização das atividades de vida diária. ${ }^{5,6}$ Nesse sentido, a prática regular de atividade física tem sido recomendada por promover, dentre outros benefícios, melhora da resistência aeróbia, o que contribui para manutenção do bem-estar físico e independência funcional da população idosa.?

Nos últimos anos, as atividades físicas baseadas na interação corpo-mente vêm aumentando sua popularidade no Ocidente. ${ }^{8-11}$ O Tai Chi Chuan (TCC) é um exemplo desse tipo de atividade. Essa modalidade é considerada uma prática heterogênea que integra múltiplos componentes (i.e., eficiência musculoesquelética, controle da respiração, concentração mental, interação psicossocial e rituais). ${ }^{10,12,13}$ Além disso, é um método alternativo de exercício físico aeróbio. ${ }^{11,14}$

Estudos prévios apontam que o TCC é caracterizado como uma atividade aeróbia de moderada intensidade (50-60\% do consumo máximo de oxigênio - $\mathrm{VO}_{2}$ máx). ${ }^{15-17}$ De acordo com as diretrizes do Colégio Americano de Medicina do Esporte sobre prescrição de exercício físico para idosos, ${ }^{6}$ atividades moderadas são eficazes para melhorar a resistência aeróbia dessa população. É plausível especular que a prática regular do TCC possa promover aumento da resistência aeróbia de idosos sedentários, assim, o objetivo do presente estudo foi analisar o efeito de uma intervenção de 12 semanas de TCC sobre a resistência aeróbia de idosas sedentárias.

\section{METODOLOGIA}

\section{Desenho do Estudo}

O presente estudo é caracterizado como quaseexperimental. Dessa forma, buscou-se analisar o efeito da variável independente (prática do TCC) sobre a variável dependente (resistência aeróbia).
Fizeram parte da pesquisa 11 idosas sedentárias $\left(67,9 \pm 6,8\right.$ anos; $\left.25,9 \pm 2,8 \mathrm{~kg} / \mathrm{m}^{2}\right)$, as quais foram informadas sobre os procedimentos do estudo e assinaram um termo de consentimento livre e esclarecido para participação no mesmo, de acordo com a Resolução no 196/96 do Conselho Nacional de Saúde. O estudo foi aprovado pelo comitê de ética em pesquisa da Universidade Federal do Rio Grande do Norte (UFRN), sob o protocolo no 160/2011.

\section{Avaliação da Resistência Aeróbia}

Para avaliação da resistência aeróbia, foram realizados os testes de caminhada de seis minutos e o de marcha estacionária de dois minutos, sendo adotados os procedimentos estabelecidos por Rikli \& Jones. ${ }^{18}$ Para garantir maior validade interna do estudo, as avaliações foram conduzidas por um único avaliador nos momentos pré e pós12 semanas de prática do TCC.

\section{Prática do Tai Chi Chuan}

Os indivíduos foram submetidos a 12 semanas de prática de TCC estilo Wu, três vezes por semana. Cada sessão foi constituída de dez minutos de aquecimento, 35 minutos de técnicas específicas do Wu TCC e cinco minutos de desaquecimento (volta à calma), totalizando 50 minutos. No aquecimento foram realizados alongamentos para os grandes grupamentos musculares de membros superiores e inferiores, além de exercícios de propriocepção e equilíbrio (estático e dinâmico). A sessão específica de TCC incluiu aproximadamente 80 posturas, com repetições de algumas sequências.

Nesse momento da aula, as praticantes foram instruídas a realizar os movimentos em uma intensidade moderada, com base na escala de Borg CR $10^{19}$ (percepção subjetiva do esforço entre 3-4). Além disso, as participantes foram estimuladas pelo instrutor a realizar os movimentos e posturas em velocidade padrão (lenta - moderada). O desaquecimento incluiu exercícios de respiração (ex., Chi Kung) e relaxamento (ex., Mok So). As 
voluntárias foram orientadas a não se engajar em outro programa de atividade física regular, mantendo, dessa forma, os hábitos anteriores ao início do programa de TCC.

\section{Análise Estatística}

Os dados apresentaram distribuição normal, verificada por meio do teste Shapiro-Wilk. Os resultados estão expressos em média e desviopadrão. Para análise inferencial (pré e pós- intervenção) foi utilizado o teste $t$ de Student pareado. Adicionalmente, a magnitude do efeito da intervenção (prática de TCC) sobre as variáveis dependentes do estudo (desempenho no teste de marcha estacionária de dois minutos e teste de caminhada de seis minutos) foi calculada por meio do $d$ de Cohen. Valores entre 0,20-0,49; 0,50-0,79 e maior ou igual a 0,80 foram utilizados para determinar, respectivamente, efeito de baixa, moderada e alta magnitude. ${ }^{20} \mathrm{O}$ pacote estatístico SPSS $^{\circledR}$ versão 19.0 foi utilizado, sendo adotado um $p<0,05$ como significância estatística.
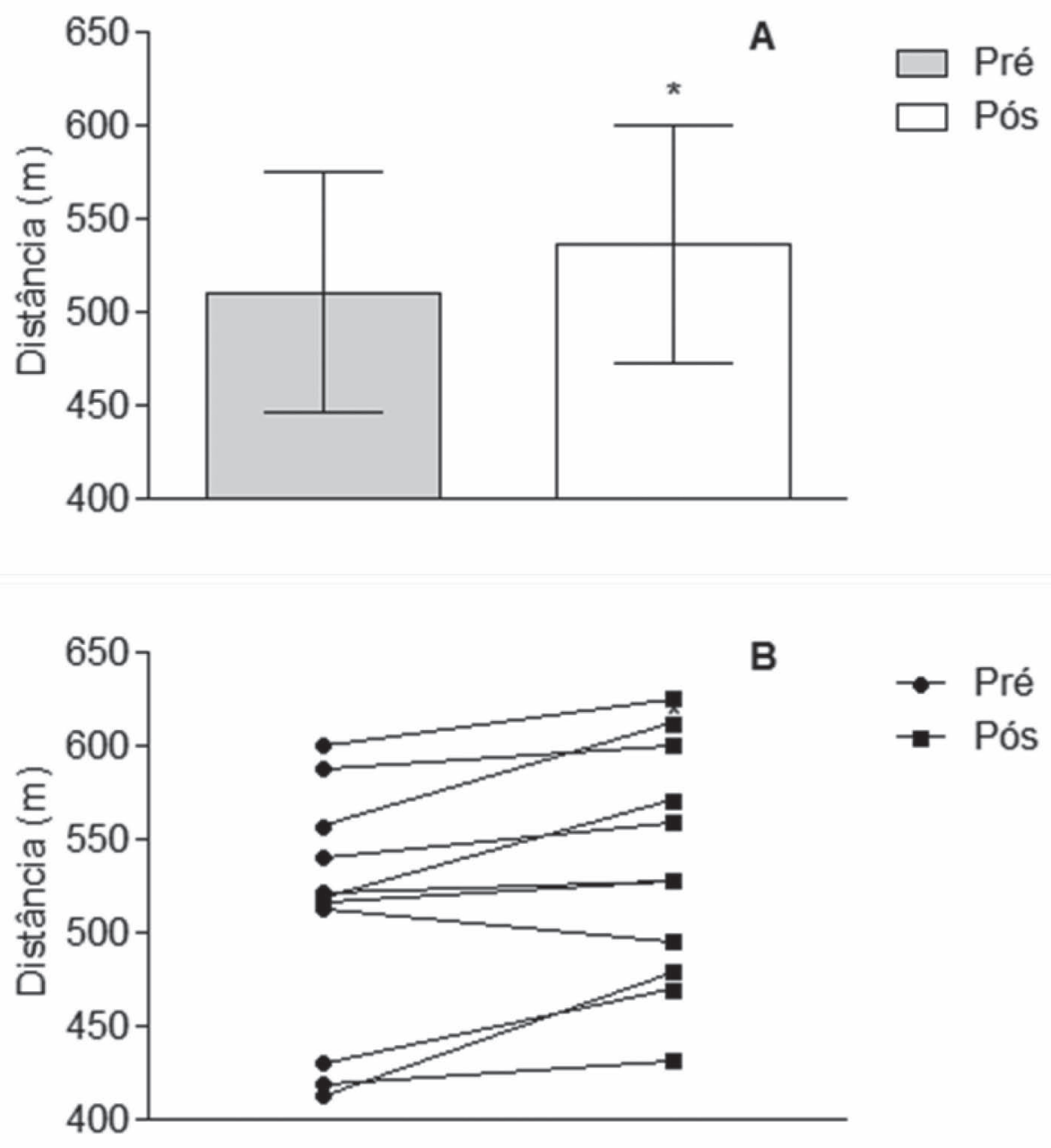

Teste de Caminhada de 6 Minutos

Figura 1 - Desempenho no teste de caminhada de seis minutos pré e pós 12 semanas de prática de Tai Chi Chuan $(\mathrm{n}=11)$. 

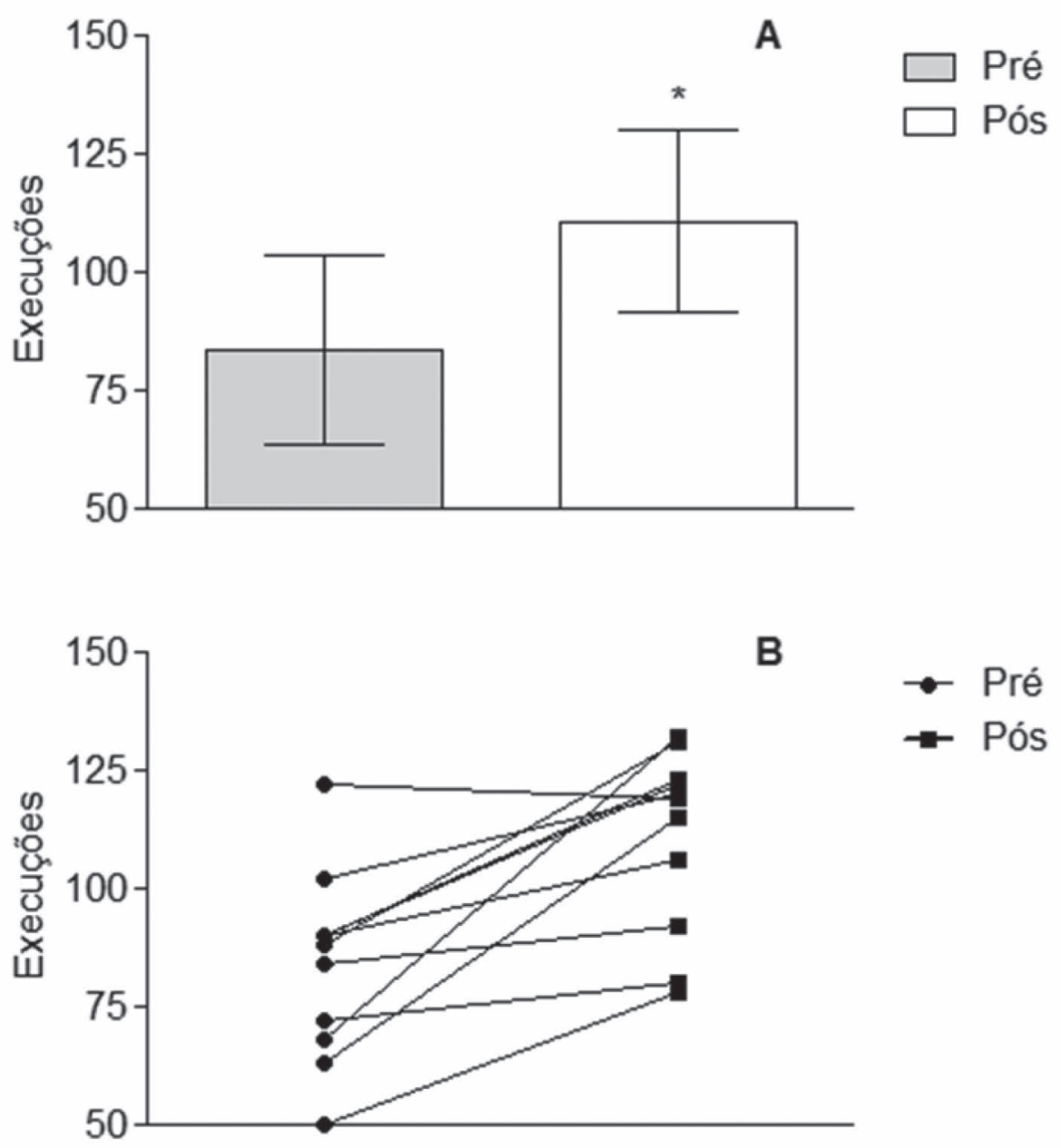

\section{Marcha Estacionária}

Figura 2 - Desempenho no teste de marcha estacionária de dois minutos pré e pós 12 semanas de prática de Tai Chi Chuan ( $\mathrm{n}=11)$.

Nota: A = resultado do grupo (expresso em média e desvio padrão); $\mathrm{B}=$ resultados individuais; $*$ = diferença estatisticamente significativa $(p<0,05)$.

\section{RESULTADOS}

Houve aumento da resistência aeróbia das idosas analisadas após 12 semanas de prática de TCC, demonstrado tanto por meio do teste de caminhada de seis minutos $(510 \pm 64$ vs. 536 \pm 63 metros; $p=0,006$ ), segundo a figura 1 , quanto no teste de marcha estacionária de dois minutos $(83 \pm 20$ vs. $110 \pm 19$ execuções; $p=$ $0,001)$, conforme a figura 2 .
$\mathrm{Na}$ análise individual, dez das 11 idosas aumentaram a distância percorrida no teste de caminhada de seis minutos (aumento médio de $5 \% ; 1,5-16 \%$ ). Da mesma forma, dez das 11 idosas realizaram mais execuções no teste de marcha estacionária de dois minutos (aumento médio de 37\%; 9,5-82,5\%).

No tocante à magnitude do efeito da intervenção sobre os testes supracitados, 
constatou-se que o TCC apresentou efeito de alta magnitude sobre o teste de marcha estacionária de dois minutos $(\mathrm{d}=1,46)$ e baixa magnitude sobre o teste de caminhada de seis minutos $(d=0,42)$.

\section{DISCUSSÃO}

O presente estudo buscou investigar o efeito da prática do TCC sobre a resistência aeróbia de idosas sedentárias. Nesse sentido, a hipótese inicial se confirmou: houve melhora da resistência aeróbia das idosas analisadas após 12 semanas de prática de TCC.

Após a intervenção, as idosas melhoraram o desempenho em ambos os testes. Entretanto, a melhora alcançada no teste de caminhada de seis minutos foi de baixa magnitude ( $\mathrm{d}=$ $0,42)$, enquanto a evolução no teste de marcha estacionária de dois minutos foi de alta magnitude (d = 1,46). É plausível especular que esse fato se deva às ações motoras específicas realizadas durante o $W u$ TCC, principalmente envolvendo membros inferiores - posturas, bases, sequência e chutes. Dessa forma, o teste de marcha estacionária de dois minutos parece ter sido mais sensível para detectar a melhora da resistência aeróbia após 12 semanas de prática de TCC, provavelmente em decorrência da maior especificidade da ação motora (princípio da especificidade).

De forma semelhante ao nosso estudo, Taylor-Piliae et al. ${ }^{14}$ analisaram o efeito do TCC sobre a resistência aeróbia de indivíduos de meia-idade e idosos. Através de estudo quaseexperimental, 39 sujeitos de ambos os sexos (66 \pm 8,3 anos) realizaram 12 semanas de Yang TCC (versão curta - 24 posturas), três vezes por semana (60 minutos por sessão). O teste de marcha estacionária de dois minutos também foi realizado para avaliar a resistência aeróbia. Os autores verificaram melhora de $12 \%$ após seis semanas ( $73 \pm 13,8$ vs. $83 \pm 18,3$ repetições) e 17,3\% após 12 semanas ( $73 \pm 13,8$ vs. 88,3 $\pm 20,2$ repetições) de intervenção.

Nossos dados corroboram os achados de Taylor-Piliae etal., ${ }^{14}$ haja vista que após 12 semanas de prática de TCC as voluntárias aumentaram em média 37\% o número de execuções no teste de marcha estacionária de dois minutos. Reiteramos que esses resultados, provavelmente, são decorrentes das ações motoras específicas do TCC, independentemente do estilo adotado.

Outros estudos confirmam a ideia de que a prática regular do TCC pode causar impacto positivo sobre a resistência aeróbia dos indivíduos. ${ }^{21,22}$ Entretanto, benefícios mais consistentes são alcançados após um longo tempo de prática (acima de um ano), especialmente em indivíduos de meia-idade e idosos previamente sedentários. $^{21} \mathrm{O}$ fato de o TCC apresentar potencial para melhoria da resistência aeróbia de idosos merece destaque, pois essa capacidade física é importante preditora de morbimortalidade por todas as causas e incapacidade nessa população. ${ }^{23-26}$ Adicionalmente, recentes ensaios clínicos controlados e randomizados, revisões sistemáticas e meta-análises têm evidenciado que o TCC é uma prática eficaz para redução de fatores de risco cardiovasculares e metabólicos, ${ }^{9-11,14,16}$ melhora da aptidão física ${ }^{9,14,27-29}$ e saúde mental ${ }^{8,9,31}$ de idosos. Além disso, apresenta alto índice de aderência entre os participantes. ${ }^{8,12,30}$

Apesar dos achados positivos, algumas limitações metodológicas, como ausência de um grupo controle e o pequeno tamanho amostral, precisam ser consideradas. Nesse sentido, os resultados do presente estudo devem ser interpretados com cautela.

\section{CONCLUSÃO}

Os resultados deste estudo evidenciam que a prática sistemática de Tai Chi Chuan (TCC) é capaz de promover melhora da resistência aeróbia de mulheres idosas previamente sedentárias. Nesse sentido, o TCC pode ser recomendado como uma modalidade de atividade aeróbia segura, de fácil aplicabilidade, baixo custo e eficaz para manutenção e melhora da saúde de idosos. Dessa forma, apresenta potencial para ser implementada em programas públicos de condicionamento físico voltados para saúde. 


\section{AGRADECEMOS}

Aos participantes e voluntários do projeto "Práticas Corporais Orientais: Capacidade
Funcional e Qualidade de Vida de Idosos", da Universidade Federal do Rio Grande do Norte. Agradecemos também à Cardioclínica Dr. Ovídio Fernandes, pelo apoio e parceria.

\section{REFERÊNCIAS}

1. Rosen MJ, Sorkin JD, Goldeberg AP, et al. Predictors of age-associated decline in maximal aerobic capacity: a comparison of four statistical models. J Appl Physiol. 1998;84(6):2163-70.

2. Fitzgerald MD, Tanaka H, Tran ZV, et al. Agerelated declines in maximal aerobic capacity in regularly exercising vs. sedentary women: a metaanalysis. J Appl Physiol. 1997;83(1):160-5.

3. Hollenberg M, Yang J, Haight TJ, Tager IB. Longitudinal changes in aerobic capacity: implications for concepts of aging. J Gerontol A Biol Sci Med Sci. 2006;61(8):851-8.

4. Fleg JL, Morrell CH, Bos AG, Brant LJ, Talbot LA, Wright JG,et al. Accelerated longitudinal decline of aerobic capacity in healthy older adults. Circulation. 2005;112(5):674-82.

5. de Vreede PL, Samson MM, van Meeteren NL,et al. Functional-task exercise versus resistance strength exercise to improve daily function in older women: a randomized, controlled trial. J Am Geriatr Soc. 2005;53(1):2-10.

6. American College of Sports Medicine, ChodzkoZajko WJ, Proctor DN, Fiatarone Singh MA, Minson CT, Nigg CR, et al. American College of Sports Medicine position stand. Exercise and physical activity for older adults. Med Sci Sports Exerc. 2009;41(7):1510-30.

7. de Souza Santos CA, Dantas EEm, Moreira MHR. Correlation of physical aptitude; functional capacity, corporal balance and quality of life (QoL) among elderly women submitted to a post-menopausal physical activities program. Arch Gerontol Geriatr. 2011;53(3):344-9.

8. Wang C, Bannuru R, Ramel J, KIupelmick B, Scott T,et al. Tai Chi on psychological wellbeing: systematic review and meta-analysis. BMC Complement Altern Med. 2010;10:23.

9. Rogers CE, Larkey LK, Keller C. A review of clinical trials of tai chi and qigong in older adults. West $\mathrm{J}$ Nurs Res. 2009;31(2):245-79.

10. Yeh GY, Wang C, Wayne P, Phillips R. Tai chi exercise for patients with cardiovascular conditions and risk factors: a systematic review. J Cardiopulm Rehabil Prev. 2009;29(3):152-60.

11. Yeh GY, Wang C, Wayne PM, Phillips RS. The effect of Tai Chi exercise on blood pressure: a systematic review. Prev Cardiol. 2008;11(2):82-9.

12. Wayne PM, Kaptchuk TJ. Challenges inherent to Tai Chi research: part I - Tai Chi as a complex multicomponent intervention. J Altern Complement Med. 2008;14(1):95-102.

13. Wayne PM, Kaptchuk TJ. Challenges inherent to T'ai Chi research: part II - Defining the intervention and optimal study design. J Altern Complement Med. 2008;14(2):191-197.

14. Taylor-Piliae RE, Haskell WL, Froelicher ES. Hemodynamic responses to a community-based Tai Chi exercise intervention in ethnic Chinese adults with cardiovascular disease risk factors. Eur J Cardiovasc Nurs. 2006;5(2):165-74.

15. Lan C, Chen SY, Lai JS. Relative exercise intensity of Tai Chi Chuan is similar in different ages and gender. Am J Chin Med. 2004;32(1):151-60.

16. Lan C, Chen SY, Lai JS. Changes of aerobic capacity, fat ratio and flexibility in older TCC practitioners: a five-year follow-up. Am J Chin Med. 2008;36(6):1041-50.

17. Lan $\mathrm{C}$, et al. Heart rate responses and oxygen consumption during Tai Chi Chuan practice. Am J Chin Med. 2001;29(3-4):403-10.

18. Rikli RE, Jones CJ. Development and validation of a functional fitness test for community-residing older adults. JAPA. 1999;7(1):129-161.

19. Borg G. Borg's perceived exertion and pain scales. Champaign, IL: Human Kinetics, 1998.

20. Cohen J. Statistical power analysis for the behavioral sciences. Hillsdale: Lawrence Erlbaum Associates; 1988.

21. Taylor-Piliae RE. The effectiveness of Tai Chi exercise in improving aerobic capacity: an updated meta-analysis. Med Sport Sci. 2008;52:40-53. 
22. Taylor-Piliae RE, Froelicher ES. Effectiveness of Tai Chi exercise in improving aerobic capacity: a metaanalysis. J Cardiovasc Nurs. 2004;19(1):48-57.

23. Lee CD, et al. Cardiorespiratory fitness, body composition, and all-cause and cardiovascular disease mortality in men. Am J Clin Nutr. 1999;69(3):373-80.

24. Laukkanen JA, et al. Cardiovascular fitness as a predictor of mortality in men. Arch Intern Med 2001;161(6):825-31.

25. Sui X, et al. Cardiorespiratory fitness and adiposity as mortality predictors in older adults. JAMA. 2007;298(21):2507-2516.

26. Carnethon MR, et al. Cardiorespiratory fitness in young adulthood and the development of cardiovascular disease risk factors. JAMA. 2003;290(23):3092-100.

27. Xu DQ, et al. Tai Chi exercise and muscle strength and endurance in older people. Med Sports Sci. 2008;52:20-9.

28. Li JX, et al. Changes in muscle strength, endurance, and reaction of the lower extremities with Tai Chi intervention. J Biomech. 2009;42:967-71.

29. Ni GX, et al. Tai chi improves physical function in older Chinese women with knee osteoarthritis. J Clin Rheumatol. 2010;16 (2):64-7.

30. Lee LY, et al. The psychosocial effect of Tai Chi on nursing home residents. J Clin Nurs. 2010;19(78):927-38.

Recebido: 08/11/2011

Revisado: 05/7/2012

Aprovado: 02/8/2012 